OPEN ACCESS

Edited by:

Susan Christina Welburn, University of Edinburgh,

United Kingdom

Reviewed by:

Konstantinos Thomas,

University General Hospital

Attikon, Greece

Eirini Christaki,

University of Cyprus, Cyprus

*Correspondence:

Maximilian F. Konig

konig@jhmi.edu

Todd H. Wagner

twagner@stanford.edu

tThese authors share senior authorship

Specialty section: This article was submitted to Infectious Diseases - Surveillance,

Prevention and Treatment,

a section of the journal

Frontiers in Medicine

Received: 04 December 2020

Accepted: 25 February 2021

Published: 31 March 2021

Citation:

Rose L, Graham L, Koenecke A Powell M, Xiong R, Shen Z, Mench B, Kinzler KW, Bettegowda C, Vogelstein B, Athey S, Vogelstein JT, Konig MF and Wagner TH (2021) The

Association Between Alpha-1 Adrenergic Receptor Antagonists and In-Hospital Mortality From COVID-19.

Front. Med. 8:637647.

doi: 10.3389/fmed.2021.637647

\section{The Association Between Alpha-1 Adrenergic Receptor Antagonists and In-Hospital Mortality From COVID-19}

Liam Rose ${ }^{1}$, Laura Graham ${ }^{1}$, Allison Koenecke ${ }^{2}$, Michael Powell ${ }^{3}$, Ruoxuan Xiong ${ }^{4}$, Zhu Shen ${ }^{5}$, Brett Mench ${ }^{3}$, Kenneth W. Kinzler ${ }^{6}$, Chetan Bettegowda ${ }^{6,7}$, Bert Vogelstein ${ }^{6}$, Susan Athey ${ }^{8}$, Joshua T. Vogelstein ${ }^{3,9}$, Maximilian F. Konig ${ }^{6,10+}$ and Todd H. Wagner ${ }^{1,11 \times+}$

${ }^{1}$ Department of Veterans Affairs Health Economics Resource Center, Palo Alto VA, Menlo Park, CA, United States, ${ }^{2}$ Institute for Computational and Mathematical Engineering, Stanford University, Stanford, CA, United States, ${ }^{3}$ Department of Biomedical Engineering, Institute for Computational Medicine, The Johns Hopkins University, Baltimore, MD, United States, ${ }^{4}$ Department of Management Science and Engineering, Stanford University, Stanford, CA, United States, ${ }^{5}$ Department of Statistics, Stanford University, Stanford, CA, United States, ${ }^{6}$ Lustgarten Laboratory, Ludwig Center, Howard Hughes Medical Institute at The Johns Hopkins Kimmel Cancer Center, Baltimore, MD, United States, ${ }^{7}$ Department of Neurosurgery and Oncology, The Johns Hopkins University School of Medicine, Baltimore, MD, United States, ${ }^{8}$ Stanford Graduate School of Business, Stanford University, Stanford, CA, United States, ${ }^{9}$ Department of Biostatistics, The Johns Hopkins Bloomberg School of Public Health at The Johns Hopkins University, Baltimore, MD, United States, ${ }^{10}$ Division of Rheumatology, Department of Medicine, The Johns Hopkins University School of Medicine, Baltimore, MD, United States, ${ }^{11}$ Department of Surgery, Stanford University, Stanford, CA, United States

Effective therapies for coronavirus disease 2019 (COVID-19) are urgently needed, and pre-clinical data suggest alpha-1 adrenergic receptor antagonists ( $\alpha_{1}$-AR antagonists) may be effective in reducing mortality related to hyperinflammation independent of etiology. Using a retrospective cohort design with patients in the Department of Veterans Affairs healthcare system, we use doubly robust regression and matching to estimate the association between baseline use of $\alpha_{1}$-AR antagonists and likelihood of death due to COVID-19 during hospitalization. Having an active prescription for any $\alpha_{1}$-AR antagonist (tamsulosin, silodosin, prazosin, terazosin, doxazosin, or alfuzosin) at the time of admission had a significant negative association with in-hospital mortality (relative risk reduction $18 \%$; odds ratio 0.73 ; $95 \% \mathrm{Cl} 0.63-0.85 ; p \leq 0.001$ ) and death within 28 days of admission (relative risk reduction $17 \%$; odds ratio $0.74 ; 95 \% \mathrm{Cl} 0.65-0.84 ; p \leq 0.001$ ). In a subset of patients on doxazosin specifically, an inhibitor of all three alpha- 1 adrenergic receptors, we observed a relative risk reduction for death of $74 \%$ (odds ratio 0.23; $95 \%$ Cl 0.03-0.94; $p=0.028$ ) compared to matched controls not on any $\alpha_{1}$-AR antagonist at the time of admission. These findings suggest that use of $\alpha_{1}$-AR antagonists may reduce mortality in COVID-19, supporting the need for randomized, placebo-controlled clinical trials in patients with early symptomatic infection.

Keywords: COVID-19, coronavirus disease, alpha-1-adrenergic receptor antagonist, infectious disease, off-label drug use 


\section{INTRODUCTION}

The viral replication phase in Coronavirus disease 2019 (COVID-19) can be followed by a hyperinflammatory host immune response, hereafter referred to as COVID-19-associated hyperinflammation, which can lead to acute respiratory distress syndrome (ARDS), multiorgan dysfunction, and death despite maximal supportive care (1-4). While dexamethasone and other immunosuppressive strategies have shown some promise in improving outcomes in patients with severe COVID-19, they have not shown benefit (and may be detrimental) when given to patients with less advanced disease (5-7). To date, immunomodulatory therapeutic strategies that prevent the development of hyperinflammation and thereby halt progression to severe COVID-19 do not exist.

Catecholamines (adrenaline, noradrenaline, and dopamine) are monoamine hormones that signal through adrenergic receptors (ARs) expressed on tissues including cells of the immune system (8-10). Cells of the innate and adaptive immune system (phagocytes, lymphocytes) are capable of producing catecholamines de novo and signal in an autocrine/paracrine self-regulatory fashion $(9,11)$. Beyond their well-established role in neurotransmission and physiological fight-or-flight responses, catecholamines have been shown to amplify immune responses and enhance acute inflammatory injury in vitro and in vivo by increasing cytokine production in immune cells (e.g., IL-6, TNF$\alpha$, MIP-2) $(8,10-12)$. In animal models of hyperinflammation, prophylactic treatment with an alpha-1 adrenergic receptor $\left(\alpha_{1}\right.$ AR) antagonist that inhibits all three receptor subtypes $\left(\alpha_{1 A^{-}}\right.$ ,$\alpha_{1 D^{-}}$, and $\left.\alpha_{1 B}-A R\right)$ can prevent cytokine storm and death by blocking deleterious catecholamine signaling and immune responses (11). In a retrospective analysis of patients hospitalized with acute respiratory distress, patients incidentally taking any $\alpha_{1}$-AR antagonist had a $34 \%$ relative risk reduction of being mechanically ventilated and dying $(n=16,801$, odds ratio 0.70$)$ compared to non-users (13). Similarly, the risk of progression to mechanical ventilation and death was significantly reduced in a retrospective analysis of $>300,000$ patients hospitalized with pneumonia who were prescribed $\alpha_{1}$-AR antagonists prior to their index admission, suggesting that baseline inhibition of catecholamine signaling may improve clinical outcomes in acute lower respiratory tract infection or inflammation (13). We therefore hypothesized that early treatment with $\alpha_{1}$-AR antagonists can improve mortality and ameliorate disease in patients with symptomatic SARS-CoV-2 infection (14), but data demonstrating the efficacy of $\alpha_{1}$-AR antagonists in COVID-19 specifically is lacking.

The objective of this study was to examine the association of use of $\alpha_{1}$-AR antagonists with in-hospital mortality in patients with COVID-19. Here, we analyzed a large cohort of patients hospitalized at Veterans Health Administration (VA) hospitals, in whom $\alpha_{1}-\mathrm{AR}$ antagonists are commonly used to treat unrelated diseases such as benign prostatic hyperplasia $(\mathrm{BPH})$, post-traumatic stress disorder (PTSD), or arterial hypertension (15). We hypothesized that patients with COVID-19 taking $\alpha_{1}$ AR antagonists at the time of hospital admission would be less likely to die during their hospitalization.

\section{METHODS}

\section{Study Population and Variables}

We included all patients admitted to a VA hospital between February 20, 2020, and October 7, 2020 with a confirmed COVID-19 diagnosis (Figure 1). Diagnosis codes for COVID19 were identified from the Centers for Disease Control and Prevention (CDC) coding guidelines for COVID-19 $(16,17)$. The VA COVID-19 Shared Data Resource was used to identify VA patients with a SARS-CoV-2 laboratory test result (18). This data resource combines VA-specific lab results with non-VA lab results using text extraction from patient medical records. Because over $90 \%$ of $\alpha_{1}$-AR antagonist users in the analysis were older men, we excluded women to reduce unmeasured confounding unrelated to COVID-19, specifically with respect to respiratory conditions. We also excluded patients under age 45 and patients over age 85 given the strong relationship between the severity of COVID-19 and age.

An expanded sample included all patients with laboratoryconfirmed, "suspected positive," or "possible positive" COVID19 according to National COVID Cohort Collaborative (N3C) criteria (19). This Suspected COVID-19 sample excluded patients who tested negative for SARS-CoV-2. To the extent we can measure COVID-19 severity at time of admission, we find that this cohort was not operationally different from the main cohort based on vital signs at time of admission (Supplementary Figure 1).

The primary outcomes were death during the index hospitalization and death within 28 days of admission. The primary exposure variable was the use of $\alpha_{1}$-AR antagonists at the time of admission for the index hospitalization. Active prescriptions of $\alpha_{1}-\mathrm{AR}$ antagonists (tamsulosin, silodosin, prazosin, doxazosin, alfuzosin, and terazosin) were identified and defined by the patient having medication on hand on the day of the index admission, regardless of dosage. Secondary analyses examined the effect of tamsulosin (the most commonly prescribed $\alpha_{1}-\mathrm{AR}$ antagonist with selective antagonism on $\alpha_{1 \mathrm{~A}}$ and $\alpha_{1 D^{-}}$, but not $\alpha_{1 B}-A R s$ ) and doxazosin (a non-selective antagonist acting on all three $\alpha_{1}$-ARs) individually. Finally, with in-hospital therapies evolving during the pandemic, we repeated the analysis by week and VA hospital to ensure results were not driven by any particular time or location.

We obtained data on patient demographics, vital signs, and prescription drugs from the VA's corporate data warehouse (CDW). Patient comorbidities were captured based on the International Classification of Diseases, Version 10 codes from VA care in the year prior to index admission. Other physiologic variables, including oxygen saturation and temperature, were defined at time of inpatient admission.

\section{Analysis}

Analyses followed the methodology of a companion paper examining patients with acute respiratory distress and pneumonia (13). Unadjusted analysis compared patients with $\alpha_{1}-\mathrm{AR}$ antagonist prescriptions to all other patients with COVID-19 using Fisher's exact-test. We then estimated propensity scores and trimmed the sample to ensure overlap in 


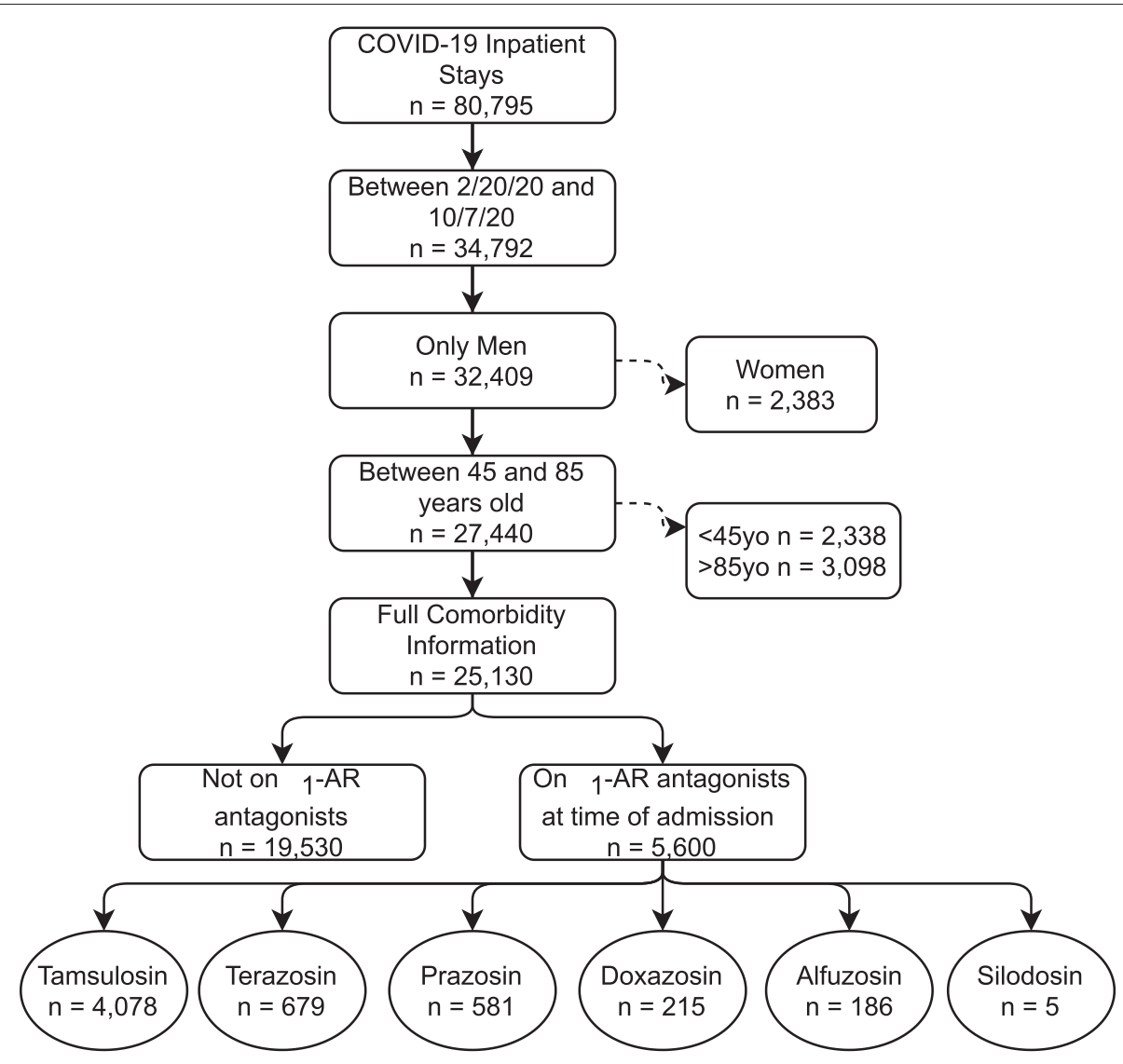

FIGURE 1 | CONSORT Flow Diagram. Consort diagram. Note that the bottom row of medications are not mutually exclusive, with a small number of patients having more than one on hand at time of admission.

the propensity score distributions of the exposed and unexposed groups. On this reduced sample, the adjusted analysis used inverse propensity-weighted logistic regression adjusting for patient age at admission (input as a demeaned cubic polynomial to allow a non-linear relationship), calendar week, location of hospitalization, and comorbidities diagnosed any time in the two years prior to the index inpatient stay. This approach is "doubly robust" in that it uses the observed confounders in both the calculation of the propensity score and the odds ratios. Comorbidities included in the matching procedure were diabetes mellitus, arterial hypertension, heart failure, ischemic heart disease, acute myocardial infarction, chronic obstructive pulmonary disease (COPD), end-stage renal disease (ESRD), and PTSD. We also included an indicator variable for oxygen saturation under 94 percent on the day of admission.

All of the control variables reflect information on patients prior to admission with COVID-19. As noted above, we controlled for secular changes in COVID-19 care using calendar week, starting with February 20, 2020. We chose not to examine endpoints during the hospital stay, such as use of a ventilator or admission to the ICU, given this is based on physician coding or data structures that we cannot assure were handled uniformly, especially during surges. We also chose not to control for processes of care during the stay given this could introduce bias in the analysis.

We then conducted a 5:1 matched analysis using the same covariates as the adjusted model (10). This approach assigns each exposed patient to a set of five unexposed patients most similar on observed characteristics and does not make assumptions about the functional form of the potential relationship between confounders and the outcome. Matches were selected using a greedy, nearest-neighbor approach based on Mahalanobis distance (11). The matched analysis used the Cochran-MantelHaenszel test to obtain odds ratios, confidence intervals, and $p$-values. We also present relative risk reductions (RRR) for the matched cohorts, and the pre- and post-matching balance of covariates is shown in Figure 2.

\section{RESULTS}

\section{Sample Characteristics}

The sample contained 25,130 patients with COVID-19, with 5,600 patients taking any $\alpha_{1}-\mathrm{AR}$ antagonist at time of admission. Of those taking $\alpha_{1}-\mathrm{AR}$ antagonists, $73 \%$ of patients were on tamsulosin $(n=4,078), 12 \%$ on terazosin $(n=679), 10 \%$ on prazosin $(n=581), 4 \%$ on doxazosin $(n=215), 3 \%$ on 


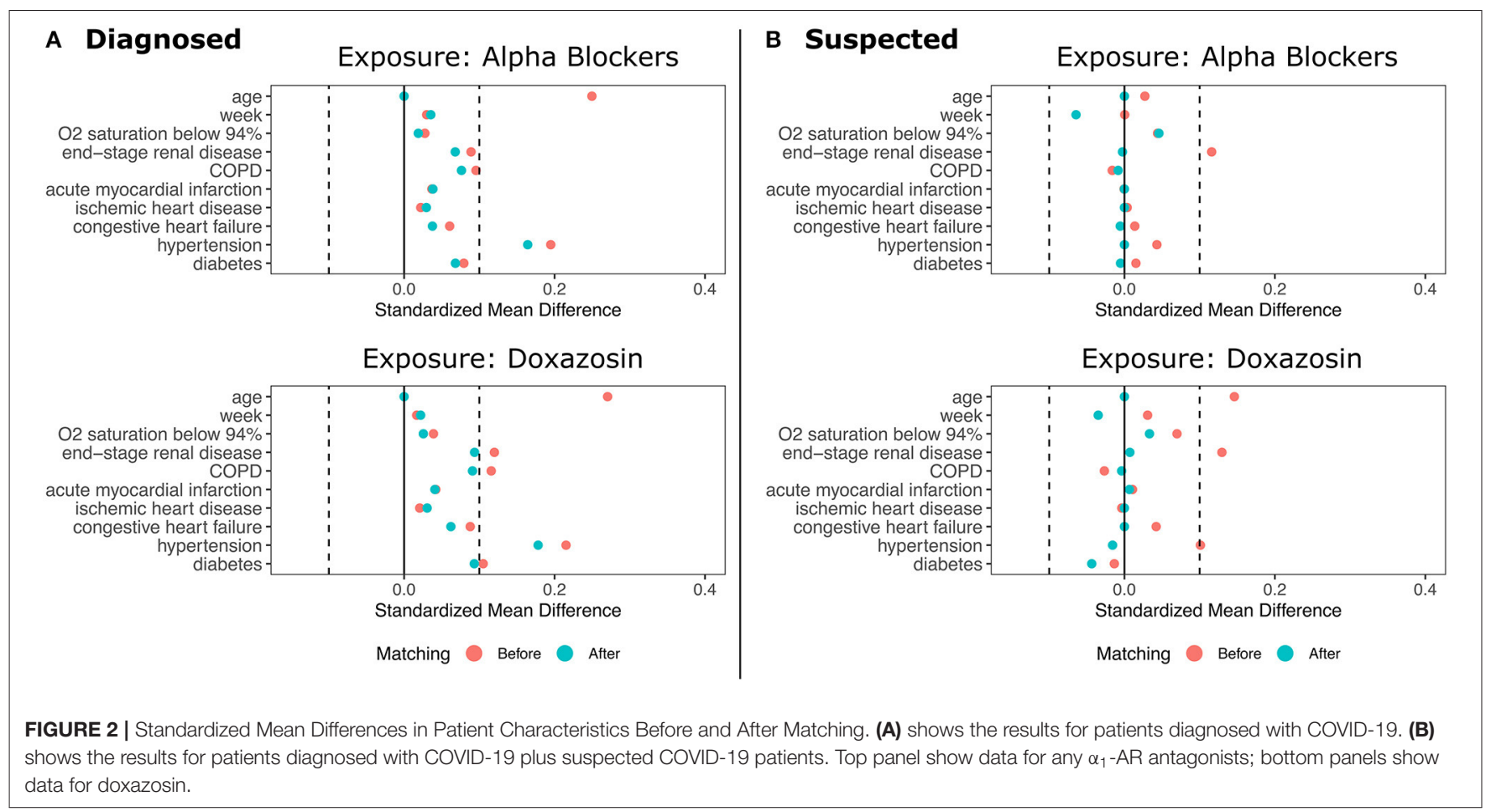

alfuzosin $(n=186)$, and $<1 \%$ were on silodosin $(n=5)$ (Figure 1). One hundred and seventy-seven patients had active prescriptions for more than one $\alpha_{1}$-AR antagonist at the time of admission. Demographic characteristics, medical comorbidities, and Charlson Comorbidity Index for patient groups prior to matching are shown in Table 1. The differences in sample characteristics after matching are summarized in Figure 2.

\section{Risk of In-Hospital and 28-Day Mortality}

For all patients admitted to VA hospitals between February 20, 2020 , and October 7,2020, the overall in-hospital mortality rate was $2.5 \%$. Among hospitalized patients with confirmed COVID19 (8.9\% of all admissions), in-hospital mortality was $6 \%$ overall and $5.5 \%$ in our sample. Patients with confirmed COVID-19 taking any $\alpha_{1}$-AR antagonist, compared to non-users, had an $18 \%$ relative risk reduction for death during their hospitalization $(243 / 5,309=4.6 \%$ in matched treatment group vs. $984 / 17,538$ $=5.6 \%$ in matched control group, $p \leq 0.001$, Figure 3 ) and a $17 \%$ relative risk reduction for death within 28 days from the date of admission $(331 / 5,309=6.2 \%$ in matched treatment group vs. $1,318 / 17,538=7.5 \%$ in matched control group, $p \leq 0.001$, Figure 3).

The top panel of Figure 3 shows the unadjusted, propensity score adjusted, and matched odds ratios among patients diagnosed with COVID-19 $(n=25,130)$. The bottom panel expands the denominator to also include patients with suspected COVID-19 $(n=32,016)$. The dark green odds ratios in Figure 3 represent all $\alpha_{1}$-AR antagonists, while the lighter green represent doxazosin. Results were similar for the suspected COVID19 sample. Patients taking any $\alpha_{1}$-AR antagonists, compared to non-users, had an $20 \%$ relative risk reduction for death $(p \leq 0.001)$ in this cohort (Figure 3).
The use of doxazosin, a non-selective $\alpha_{1}$-AR antagonist targeting all three $\alpha_{1}$-AR subtypes, resulted in a $74 \%$ relative risk reduction for death in hospitalized patients with COVID-19 during the index admission $(2 / 155=1.3 \%$ in matched treatment group vs. $39 / 775=5.0 \%$ in matched control group, odds ratio for death $0.23 ; p=0.028$, Figure 3). Use of tamsulosin, the most commonly prescribed $\alpha_{1}$-AR antagonist in this cohort with selectivity for $\alpha_{1 A^{-}}$and $\alpha_{1 D^{-}}$ARs, was associated with a $18 \%$ relative risk reduction for death during the inpatient stay (odds ratio for death 0.77; $p=0.002$, Supplementary Figure 2). Even though COVID-19 has affected different parts of the United States at different times, we found no evidence that these results were driven by any particular time period or location (Supplementary Figures 3, 4).

\section{DISCUSSION}

In this retrospective analysis of patients with COVID-19, we found a significant negative association between the use of $\alpha_{1}$ AR antagonists and in-hospital or 28-day mortality. These results are consistent with findings from a recent retrospective study of $>300,000$ patients hospitalized with pneumonia or ARDS unrelated to SARS-CoV-2 infection that identified a significant risk reduction for the progression to mechanical ventilation and death in individuals who were receiving any $\alpha_{1}$-AR antagonists as compared to non-users (5), suggesting that the benefits of $\alpha_{1}$-AR inhibition for mortality may be independent of etiology in patients with lower respiratory tract infection or inflammation.

Interestingly, we found much larger effect sizes in reducing mortality for patients treated with doxazosin, an antagonist on all three $\alpha_{1}$-AR subtypes $\left(\alpha_{1 \mathrm{~A}^{-}}, \alpha_{1 \mathrm{D}^{-}}\right.$, and $\left.\alpha_{1 \mathrm{~B}}-\mathrm{AR}\right)$, than for a 
TABLE 1 | Patient and sample characteristics at time of admission.

\begin{tabular}{|c|c|c|c|}
\hline & Control $(n=19,316)$ & Any $1 \alpha$-AR antagonist $(n=5,600)$ & Overall $(n=25,130)$ \\
\hline \multicolumn{4}{|l|}{ Age } \\
\hline Mean (SD) & $67.4(9.02)$ & $70.4(7.83)$ & $68.1(8.85)$ \\
\hline Median (Min, Max) & $69.0(45.0,85.0)$ & $72.0(45.0,85.0)$ & $70.0(45.0,85.0)$ \\
\hline \multicolumn{4}{|l|}{ Comorbidities in the prior year } \\
\hline Hypertension: $n$ (\%) & 15,603 (79.9\%) & $4,955(88.5 \%)$ & $20,558(81.8 \%)$ \\
\hline CAD: $n(\%)$ & $776(4.0 \%)$ & $283(5.1 \%)$ & $1,059(4.2 \%)$ \\
\hline CHF: $n(\%)$ & $5,611(28.7 \%)$ & $1,866(33.3 \%)$ & 7,477 (29.7\%) \\
\hline COPD: $n(\%)$ & $6,495(33.2 \%)$ & $2,284(40.8 \%)$ & $8,779(34.9 \%)$ \\
\hline Diabetes: $n(\%)$ & $9,695(49.6 \%)$ & $3,076(54.9 \%)$ & $12,771(50.8 \%)$ \\
\hline Ml: $n(\%)$ & $1,347(6.9 \%)$ & 448 (8.0\%) & $1,795(7.1 \%)$ \\
\hline $\mathrm{BPH}: n(\%)$ & $4,989(25.5 \%)$ & $4,412(78.8 \%)$ & $9,401(37.4 \%)$ \\
\hline PTSD: $n(\%)$ & $4,199(21.5 \%)$ & $1,661(29.7 \%)$ & $5,860(23.3 \%)$ \\
\hline ESRD: $n(\%)$ & $5,902(30.4 \%)$ & $2,063(37.1 \%)$ & $7,965(31.9 \%)$ \\
\hline Charlson Comorbidity Index: mean (SD) & $4.00(3.45)$ & $4.87(3.53)$ & $4.47(3.48)$ \\
\hline $\mathrm{SpO}_{2}<94 \%: n(\%)$ & $5,770(29.5 \%)$ & $1,706(30.5 \%)$ & $7,476(29.7 \%)$ \\
\hline \multicolumn{4}{|l|}{ VA Hospital } \\
\hline 508 (Atlanta, GA) & $390(2.0 \%)$ & $113(2.0 \%)$ & $503(2.0 \%)$ \\
\hline 528 (VA Upstate New York, NY) & $346(1.8 \%)$ & $100(1.8 \%)$ & $446(1.8 \%)$ \\
\hline 541 (Cleveland, OH) & $335(1.7 \%)$ & $91(1.6 \%)$ & $426(1.7 \%)$ \\
\hline 549 (Dallas, TX) & $393(2.0 \%)$ & $136(2.4 \%)$ & $527(2.1 \%)$ \\
\hline 573 (Gainesville, FL) & $384(2.0 \%)$ & $134(2.4 \%)$ & $518(2.1 \%)$ \\
\hline 580 (Houston, TX) & $525(2.7 \%)$ & $175(3.1 \%)$ & $700(2.8 \%)$ \\
\hline 589 (Kansas City, MO) & $421(2.2 \%)$ & $171(3.1 \%)$ & $592(2.3 \%)$ \\
\hline 614 (Memphis, TN) & 827 (4.2\%) & $264(4.7 \%)$ & $1,092(4.3 \%)$ \\
\hline 626 (Nashville, TN) & $471(2.4 \%)$ & $131(2.4 \%)$ & $602(2.4 \%)$ \\
\hline 630 (VA New York Harbor, NY) & $458(2.3 \%)$ & $104(1.9 \%)$ & $562(2.2 \%)$ \\
\hline 636 (Omaha, NE) & $286(1.4 \%)$ & $88(1.6 \%)$ & $374(1.5 \%)$ \\
\hline 644 (Phoenix, AZ) & $413(2.1 \%)$ & 105 (1.9\%) & $518(2.1 \%)$ \\
\hline 657 (St Louis, MO) & $388(2.0 \%)$ & $94(1.7 \%)$ & $482(1.9 \%)$ \\
\hline 671 (San Antonio, TX) & 509 (2.6\%) & 119 (2.1\%) & $628(2.5 \%)$ \\
\hline 673 (Tampa, FL) & $413(2.1 \%)$ & $117(2.1 \%)$ & $530(2.1 \%)$ \\
\hline Other VA hospitals & $12,973(66.4 \%)$ & $3,657(65.2 \%)$ & 16,630 (66.1\%) \\
\hline
\end{tabular}

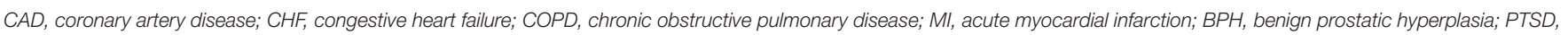

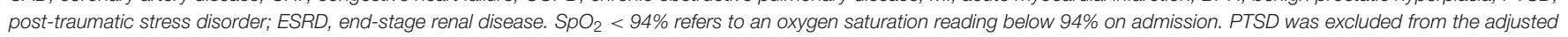
analysis due to collinearity with other comorbidities. Listed VA hospitals had the most COVID-19 inpatient hospitalizations during the study period.

pooled population of patients treated with any $\alpha_{1}-\mathrm{AR}$ antagonist in whom tamsulosin was the most common drug (72\%). This was similarly true for patients treated exclusively with tamsulosin, a "uroselective" $\alpha_{1}$-AR antagonist on $\alpha_{1 A^{-}}$and $\alpha_{1 D^{-}}$ARs without clinically relevant inhibition of $\alpha_{1 B}$-ARs expressed by immune cells and the peripheral vasculature (20). In patients with testconfirmed COVID-19, baseline use of doxazosin was associated with significantly reduced in-hospital and 28-day mortality compared to controls (odds ratio for death during admission 0.19 in adjusted cohort; odds ratio and relative risk reduction for death 0.23 and $74 \%$ in matched cohort, respectively). Baseline use of tamsulosin in patients with confirmed COVID-19, by comparison, was associated with significant, but less pronounced reductions in mortality. A similar trend was previously observed in patients with pneumonia in whom use of doxazosin was associated with lower risk of mechanical ventilation and death than tamsulosin (13). These observed differences in effect size are biologically plausible and may reflect the distinct pharmacological selectivity of doxazosin and tamsulosin for $\alpha_{1^{-}}$ AR subtypes.

Immune cells can induce expression of all three $\alpha_{1}$-AR subtypes (i.e., $\alpha_{1 \mathrm{~A}^{-}}, \alpha_{1 \mathrm{D}^{-}}$, and $\alpha_{1 \mathrm{~B}^{-}}$ARs (21), and catecholamine signaling through these individual receptors may be highly redundant (12). As such, $\alpha_{1}-\mathrm{AR}$ antagonists acting on all three receptor subtypes (i.e., doxazosin, prazosin, alfuzosin, terazosin) may be required to effectively interrupt autocrine and paracrine catecholamine signaling in monocytes and other immune cells that enhance inflammatory injury $(14,20)$. Indeed, pre-clinical data suggests that non-selective $\alpha_{1}$-AR antagonists are effective in preventing hyperinflammation and death in animal models of cytokine storm syndrome (11). The markedly improved survival in patients on doxazosin as compared to 


\section{Department of Veterans Affairs: Mortality all $a_{1}-A R$ antagonists |doxazosin}

\section{Diagnosed COVID-19 $(n=25,130)$}

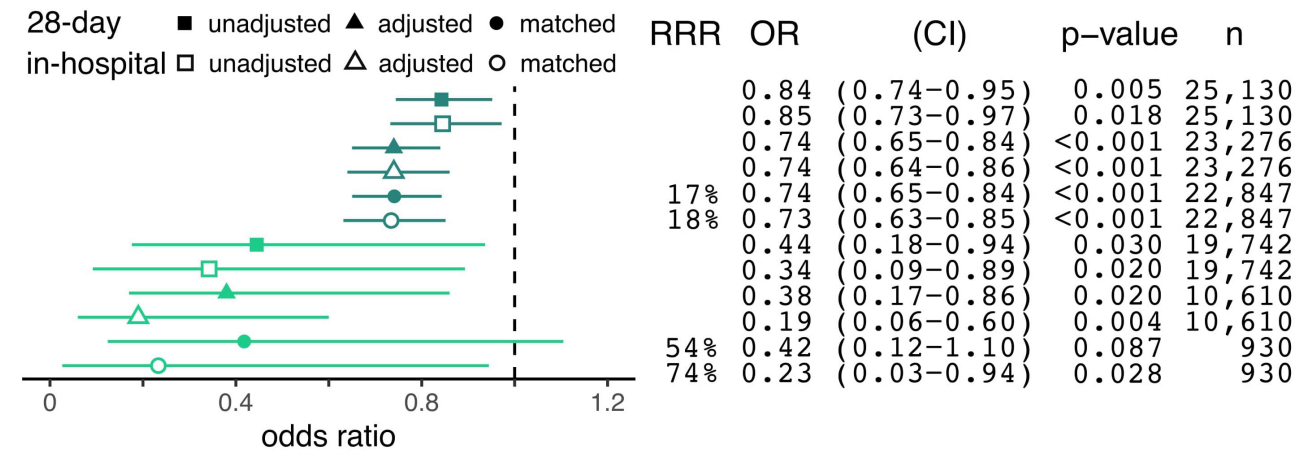

\section{Suspected COVID-19 $(n=32,016)$}

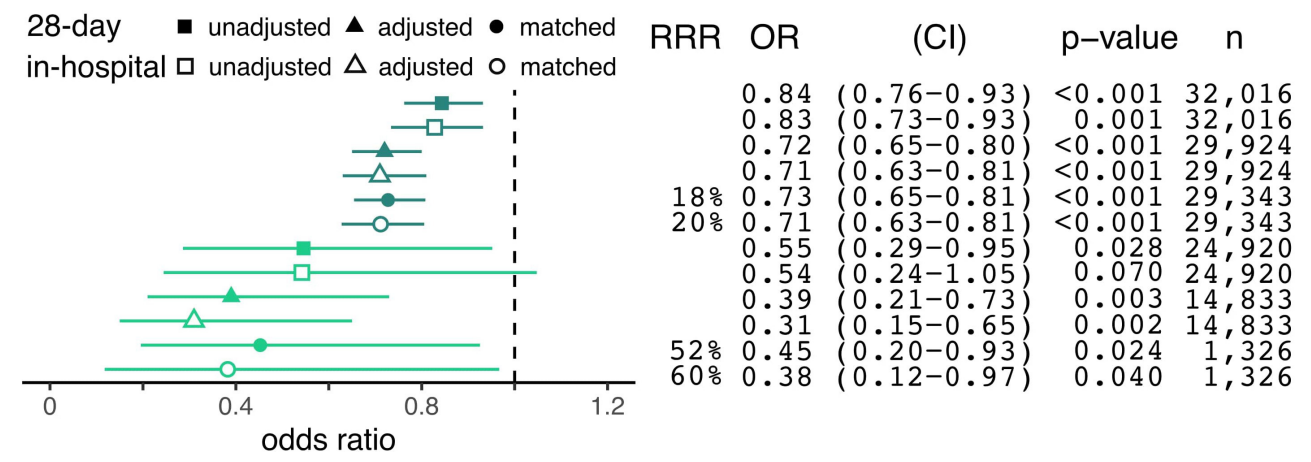

FIGURE 3 | The Association Between Alpha-1 Adrenergic Receptor Antagonists and In-Hospital and 28-Day Mortality from COVID-19. Data are shown for hospitalized patients with confirmed COVID-19 (top panel) and with confirmed plus suspected COVID-19 (e.g., no confirmatory testing available, bottom panel). Forest plots show the odds ratios (OR) for in-hospital mortality based on prior use of any $\alpha_{1}$-AR antagonists (i.e., tamsulosin, silodosin, prazosin, terazosin, doxazosin, or alfuzosin; dark green) or only doxazosin (light green) in each panel. Unadjusted (square), adjusted model (triangle), and matched model (circle) analyses are shown for each sample group. Filled symbols reflect the odds of death within 28 days from index hospital admission (including deaths after discharge), whereas empty symbols reflect odds of death during the index admission. Relative risk reduction (RRR), odds ratios (ORs) for death, $95 \%$ confidence intervals (Cl), $p$-values, and sample size (n) for each analysis are shown on the right.

tamsulosin or any $\alpha_{1}$-AR antagonist (a cohort highly enriched in tamsulosin use) may therefore be consistent with a redundancy in catecholamine signaling pathways which are globally inhibited by doxazosin, whereas tamsulosin allows for continued signaling through the $\alpha_{1 \mathrm{~B}}-\mathrm{AR}$. These findings have practical implications for the selection of $\alpha_{1}$-AR antagonists for the prevention of inflammatory injury and suggest that the immunomodulatory benefits may not be uncoupled from inhibition of $\alpha_{1 \mathrm{~B}}$-ARs expressed on the peripheral vasculature.

Additional studies have explored the efficacy of $\alpha_{1}$-AR blockade in the prevention of inflammatory and autoimmune injury. In a model of encephalitis, early $\alpha_{1}$-AR inhibition reversed neutrophil infiltration in lungs and prevented hemorrhagic pulmonary edema (22). The non-selective $\alpha_{1}$-AR antagonist prazosin has been shown to ameliorate experimental autoimmune encephalomyelitis (23). In a pre-clinical model of ischemia-reperfusion injury, prazosin administration resulted in decreased expression of IL-6, TNF- $\alpha$, IL-10, and IL-1, and prevented mortality (24). Finally, $\alpha_{1}$-AR antagonism has been shown to block cytokine production in human peripheral blood mononuclear cells from patients with juvenile polyarticular arthritis, and treatment with doxazosin abrogated catecholamine-augmented secretion of IL-6 (25). These studies suggest a role of catecholamine-associated augmentation of injurious cytokine responses beyond cytokine release syndrome and acute lung infection and highlights the potential of $\alpha_{1}$-AR antagonists across various inflammatory diseases.

One concern with observational analysis is confounding by indication, especially if medications given during a hospital stay are correlated with disease severity. To avoid confounding by indication, this analysis examined the use of $\alpha_{1}$-AR antagonists prior to index hospitalization. This class of medications is primarily used to manage chronic diseases such as arterial hypertension, PTSD, or $\mathrm{BPH}$. As such, prescribing practices would not be biased by the severity of COVID-19. In addition, our results were not driven by a specific location or time period. 
This study has important strengths and weaknesses. We have focused on mortality as a definitive clinical outcome, thereby avoiding process measures, such as use of mechanical ventilators or admission to an ICU, that are subject to local and individual practice patterns and would be biased if clinicians or hospitals changed their practices in unobserved ways. Another strength is our use of information prior to the COVID-19 admission for risk adjustment. One limitation in this study was the exclusion of women which was required due to limitations in samples size since $\alpha_{1}$-AR antagonists are most commonly used to treat benign prostatic hyperplasia and $90 \%$ of patients in the VA system are men (26). A second limitation, best addressed in prospective clinical trials, was our inability to examine dose effects given our sample size.

Our results suggest that inhibition of catecholamine signaling with doxazosin (and other $\alpha_{1}-\mathrm{AR}$ antagonists) may reduce in-hospital and 28-day mortality in patients with COVID19 and highlight the need for randomized placebo-controlled clinical trials to examine the efficacy of $\alpha_{1}$-AR antagonists for improving survival and preventing adverse outcomes from COVID-19. Importantly, $\alpha_{1}-\mathrm{AR}$ antagonists are inexpensive, administered orally, do not require refrigeration, and have a wellestablished safety profile. Thus, if trials confirm these results, $\alpha_{1}$-AR antagonists could be widely deployed to reduce mortality from inflammatory injury. Importantly, $\alpha_{1}$-AR antagonists are immunomodulatory, but not immunosuppressive drugs. Longterm use of doxazosin does not appear to be associated with the development of opportunistic infection in human studies (27). Indeed, some studies suggest an overall decreased risk of urinary tract infection compared to placebo as may be expected based on its effect on dynamic prostate and bladder function (28). The absence of serious infectious complications may be explained by the unique mechanism of action of $\alpha_{1}$-AR antagonists compared to immunosuppressive drugs currently employed in the treatment of severe COVID-19 (e.g., dexamethasone, baricitinib, tocilizumab) which confer an increased risk of opportunistic infection.

In summary, patients hospitalized with COVID-19 had lower odds of in-hospital and 28-day death if they had an active prescription for any $\alpha_{1}-\mathrm{AR}$ antagonist (tamsulosin, silodosin, prazosin, terazosin, doxazosin, or alfuzosin) at the time of admission. Among different $\alpha_{1}$-AR antagonists, doxazosin was associated with a $74 \%$ relative risk reduction for death, while tamsulosin had a more modest $18 \%$ relative risk reduction for death. A clinical trial testing the efficacy and safety of $\alpha_{1}$-AR antagonists such as doxazosin to prevent hyperinflammation and reduce mortality in COVID-19 would appear warranted.

\section{DATA AVAILABILITY STATEMENT}

The original contributions generated in the study are included in the article/Supplementary Material, further inquiries can be directed to the corresponding author.

\section{ETHICS STATEMENT}

The studies involving human participants were reviewed and approved by Stanford IRB. Written informed consent for participation was not required for this study in accordance with the national legislation and the institutional requirements.

\section{AUTHOR CONTRIBUTIONS}

JV, BV, CB, and MK conceived of the idea. LR, LG, AK, MP, and RX conducted statistical analyses and results presentation. AK, MP, RX, ZS, and SA developed the methodology. LR, MK, $\mathrm{JV}$, and TW wrote the manuscript with input from all authors. MK and TW are co-senior authors. All authors reviewed the final manuscript.

\section{FUNDING}

The work of AK was supported by the National Science Foundation Graduate Research Fellowship under Grant No. DGE-1656518. MK was supported by the National Institute of Arthritis and Musculoskeletal and Skin Diseases of the National Institutes of Health under Award No. T32AR048522. CB was supported by the Burroughs Wellcome Career Award for Medical Scientists. SA was supported by the Office of Naval Research under Grant N00014-17-1-2131. This work was further supported by The Virginia and D.K. Ludwig Fund for Cancer Research, The Lustgarten Foundation for Pancreatic Cancer Research, the BKI Cancer Genetics and Genomics Research Program. TW was funded by a VA Research Career Scientist Award (RCS-17-154). Research, including data analysis, was partially supported by funding from Microsoft Research and Fast Grants, part of the Emergent Ventures Program at The Mercatus Center at George Mason University.

\section{ACKNOWLEDGMENTS}

Any opinion, findings, and conclusions or recommendations expressed in this material are those of the authors and do not necessarily reflect the views of the US Department of Veterans Affairs or the National Science Foundation. This project was sponsored by the VA Office of Reporting, Analytics, Performance, Improvement and Deployment, and the VA Center for Medication Safety, and approved as quality improvement by the Stanford University Institutional Review Board.

\section{SUPPLEMENTARY MATERIAL}

The Supplementary Material for this article can be found online at: https://www.frontiersin.org/articles/10.3389/fmed. 2021.637647/full\#supplementary-material

Supplementary Figure 1 | Vital Signs at Time of Admission. The diagrams show vital signs for patients diagnosed with COVID-19 (red line) and an expanded cohort of patients with suspected COVID-19 (blue line). Smoothed lines are from a LOESS model with 95\% confidence intervals shown (gray ribbons).

Supplementary Figure 2 | In-hospital and 28-Day Mortality by Use of Tamsulosin at Time of Hospital Admission with COVID-19. Data are shown for hospitalized patients diagnosed with confirmed COVID-19 (top panel) and with confirmed plus suspected COVID-19 (bottom panel). Forest plots showing odds ratios (OR) of 
in-hospital mortality based on prior use of any alpha-1 adrenergic receptor antagonists (dark green) or tamsulosin (light blue) in each panel. Relative risk reduction (RRR), odds ratios (ORs) for death, 95\% confidence intervals (Cl), and $p$-values (for unadjusted, adjusted, and matched models), and sample size ( $n)$ for each analysis are shown on the right.

Supplementary Figure 3 | Adjusted Odds of In-hospital Mortality and Use of $\alpha_{1}$-AR Antagonists by Week. Top panel shows adjusted odds ratios of in-hospital mortality and use of $\alpha_{1}$-AR antagonists by week of admission. Top panel truncated between 0 and 2 to aid visualization. Bottom panel shows number of new admissions by week and use of $\alpha_{1}$-AR antagonists (bottom).
Supplementary Figure 4 | Adjusted Odds of In-hospital Mortality and Use of $\alpha_{1}$-AR Antagonists by VA Station. Top panel shows adjusted odds ratios of in-hospital mortality in patients taking $\alpha_{1}$-AR antagonists by VA station. Top panel truncated between 0 and 2 to aid visualization. Bottom panel shows number of new admissions and use of $\alpha_{1}-A R$ antagonists by VA station (bottom). For other VA stations, the number of admissions of patients not using $\alpha_{1}$-AR antagonists was 7,645 and number of admissions of patients using $\alpha_{1}$-AR antagonists was 1,845. VA stations shown: $508=$ Atlanta, $549=$ Dallas, $573=$ Gainesville, $580=$ Houston, $589=$ Kansas City, $614=$ Memphis, $630=$ New York Harbor, $644=$ Phoenix, $671=$ San Antonio, $673=$ Tampa.

\section{REFERENCES}

1. Manson JJ, Crooks C, Naja M, Ledlie A, Goulden B, Liddle T, et al. COVID-19-associated hyperinflammation and escalation of patient care: a retrospective longitudinal cohort study. Lancet Rheumatol. (2020) 2:e594-602. doi: 10.1016/S2665-9913(20)30275-7

2. Fajgenbaum DC, June CH. Cytokine storm. N Engl J Med. (2020) 383:2255-73. doi: 10.1056/NEJMra2026131

3. Henderson LA, Canna SW, Schulert GS, Volpi S, Lee PY, Kernan KF, et al. On the Alert for cytokine storm: immunopathology in COVID-19. Arthritis Rheumatol. (2020) 72:1059-63. doi: 10.1002/art.41285

4. Webb BJ, Peltan ID, Jensen P, Hoda D, Hunter B, Silver A, et al. Clinical criteria for COVID-19-associated hyperinflammatory syndrome: a cohort study. Lancet Rheumatol. (2020) 2:e754-63. doi: 10.1016/S2665-9913(20)30343-X

5. Recovery Collaborative Group, Horby P, Lim WS, Emberson JR, Mafham M, Bell JL, et al. Dexamethasone in hospitalized patients with covid-19-preliminary report. N Engl J Med. (2021) 384:693-704. doi: 10.1056/NEJMoa2021436

6. Salama C, Han J, Yau L, Reiss WG, Kramer B, Neidhart JD, et al. Tocilizumab in patients hospitalized with covid-19 pneumonia. N Engl J Med. (2021) 384:20-30. doi: 10.1056/NEJMoa2030340

7. Kalil AC, Patterson TF, Mehta AK, Tomashek KM, Wolfe CR, Ghazaryan V, et al. Baricitinib plus remdesivir for hospitalized adults with covid-19. N Engl J Med. (2020) 384:795-807. doi: 10.1056/NEJMoa2031994

8. Barnes MA, Carson MJ, Nair MG. Non-traditional cytokines: how catecholamines and adipokines influence macrophages in immunity, metabolism and the central nervous system. Cytokine. (2015) 72:210-9. doi: 10.1016/j.cyto.2015.01.008

9. Flierl MA, Rittirsch D, Nadeau BA, Chen AJ, Sarma JV, Zetoune FS, et al. Phagocyte-derived catecholamines enhance acute inflammatory injury. Nature. (2007) 449:721-5. doi: 10.1038/nature06185

10. Grisanti LA, Perez DM, Porter JE. Modulation of immune cell function by $\alpha(1)$-adrenergic receptor activation. Curr Top Membr. (2011) 67:113-38. doi: 10.1016/B978-0-12-384921-2.00006-9

11. Staedtke V, Bai R-Y, Kim K, Darvas M, Davila ML, Riggins GJ, et al. Disruption of a self-amplifying catecholamine loop reduces cytokine release syndrome. Nature. (2018) 564:273-7. doi: 10.1038/s41586-018-0774-y

12. Grisanti LA, Woster AP, Dahlman J, Sauter ER, Combs CK, Porter JE. $\alpha 1$-adrenergic receptors positively regulate Toll-like receptor cytokine production from human monocytes and macrophages. J Pharmacol Exp Ther. (2011) 338:648-57. doi: 10.1124/jpet.110.178012

13. Koenecke A, Powell M, Xiong R, Shen Z, Fischer N, Huq S, et al. Alpha-1 adrenergic receptor antagonists to prevent acute respiratory distress syndrome and death from cytokine storm syndrome. arXiv. (2020). arXiv:2004.10117.

14. Konig MF, Powell M, Staedtke V, Bai R-Y, Thomas DL, Fischer N, et al. Preventing cytokine storm syndrome in COVID-19 using $\alpha-1$ adrenergic receptor antagonists. J Clin Invest. (2020) 130:3345-7. doi: 10.1172/JCI1 39642

15. Raskind MA, Peterson K, Williams T, Hoff DJ, Hart K, Holmes H, et al. A trial of prazosin for combat trauma PTSD with nightmares in active-duty soldiers returned from Iraq and Afghanistan. AJP. (2013) 170:1003-10. doi: 10.1176/appi.ajp.2013.12081133

16. CDC. ICD-10-CM Official Coding Guidance Interim Advice for Coronavirus. Washington DC: CDC (2020).

17. CDC. ICD-10-CM Official Coding and Reporting Guidleines COVID-19 Guidelines. Washington DC: CDC (2020).

18. Department of Veterans Affairs COVID-19 National Summary. Available online at: https://www.accesstocare.va.gov/Healthcare/ COVID19NationalSummary (accessed January 2, 2021).

19. National Center for Advancing Translational Sciences. National COVID Cohort Collaborative Phenotype Data Acquisition. Available online at: https://github. com/National-COVID-Cohort-Collaborative/Phenotype_Data_Acquisition/ wiki/Latest-Phenotype (accessed January 2, 2021).

20. Araki T, Monden K, Araki M. Comparison of $7 \alpha(1)$-adrenoceptor antagonists in patients with lower urinary tract symptoms associated with benign prostatic hyperplasia:a short-term crossover study. Acta Med Okayama. (2013) 67:24551. doi: 10.18926/AMO/51069

21. Stuart EA. Matching methods for causal inference: a review and a look forward. Stat Sci. (2010) 25:1-21. doi: 10.1214/09-STS313

22. Lu W-H, Hsieh K-S, Lu P-J, Wu Y-S, Ho W-Y, Cheng P-W, et al. Different impacts of $\alpha$ - and $\beta$-blockers in neurogenic hypertension produced by brainstem lesions in rat. Anesthesiology. (2014) 120:1192-204. doi: 10.1097/ALN.0000000000000218

23. Brosnan CF, Goldmuntz EA, Cammer W, Factor SM, Bloom BR, Norton WT. Prazosin, an alpha 1-adrenergic receptor antagonist, suppresses experimental autoimmune encephalomyelitis in the Lewis rat. Proc Natl Acad Sci USA. (1985) 82:5915-9. doi: 10.1073/pnas.82.17.5915

24. Wang L, Xue Y, Ma H, Shi H, Wang L, Cui X. Prazosin protects myocardial cells against anoxia-reoxygenation injury via the extracellular signal-regulated kinase signaling pathway. Mol Med Rep. (2018) 17:2145-52. doi: $10.3892 / \mathrm{mmr} .2017 .8175$

25. Heijnen CJ, Rouppe van der Voort C, Wulffraat N, van der Net J, Kuis W, Kavelaars A. Functional alpha 1-adrenergic receptors on leukocytes of patients with polyarticular juvenile rheumatoid arthritis. J Neuroimmunol. (1996) 71:223-6. doi: 10.1016/S0165-5728(96)00125-7

26. US Department of Veterans Affairs, Veterans Health Administration. The Changing Face of Women Veterans. Available online at: https://www. womenshealth.va.gov/WOMENSHEALTH/latestinformation/facts.asp (accessed January 2, 2021).

27. Drug Approval Package: Cardura XL (doxazosin mesylate) NDA \#021269. Available online at: https://www.accessdata.fda.gov/drugsatfda_docs/nda/ 2005/021269_carduraxl_toc.cfm (accessed February 6, 2021).

28. McConnell JD, Roehrborn CG, Bautista OM, Andriole GL, Dixon CM, Kusek JW, et al. The long-term effect of doxazosin, finasteride, and combination therapy on the clinical progression of benign prostatic hyperplasia. $N$ Engl J Med. (2003) 349:2387-98. doi: 10.1056/NEJMoa030656

Conflict of Interest: In 2017, The Johns Hopkins University (JHU) filed a patent application on the use of various drugs to prevent cytokine release syndromes, on which BV and $\mathrm{KK}$ are listed as inventors. JHU will not assert patent rights from this filing for treatment related to COVID-19. MK received personal fees from Bristol-Myers Squibb and Celltrion, unrelated to the manuscript. BV and KK are founders of and hold equity in Thrive Earlier Detection. KK is a consultant to and is on the Board of Directors of Thrive Earlier Detection. BV and KK are founders of, hold equity in, and serve as consultants to Personal Genome 
Diagnostics. KK and BV are consultants to Sysmex, Eisai, and CAGE Pharma and hold equity in CAGE Pharma. BV is also a consultant to Nexus. KK and BV are consultants to and hold equity in NeoPhore. CB is a consultant to DepuySynthes and Bionaut Pharmaceuticals. CB, BV, and KK are also inventors on technologies unrelated or indirectly related to the work described in this article. Licenses to these technologies are or will be associated with equity or royalty payments to the inventors, as well as to JHU. The terms of all these arrangements are being managed by JHU in accordance with its conflict of interest policies. $\mathrm{SA}$ is an advisor and holds an equity stake in two private companies, Prealize (Palo Alto, California, USA) and Dr. Consulta (Brazil). Prealize is a healthcare analytics company, and Dr. Consulta operates a chain of low-cost medical clinics in Brazil.
The remaining authors declare that the research was conducted in the absence of any commercial or financial relationships that could be construed as a potential conflict of interest.

Copyright (c) 2021 Rose, Graham, Koenecke, Powell, Xiong, Shen, Mench, Kinzler, Bettegowda, Vogelstein, Athey, Vogelstein, Konig and Wagner. This is an open-access article distributed under the terms of the Creative Commons Attribution License (CC $B Y)$. The use, distribution or reproduction in other forums is permitted, provided the original author(s) and the copyright owner(s) are credited and that the original publication in this journal is cited, in accordance with accepted academic practice. No use, distribution or reproduction is permitted which does not comply with these terms. 